\title{
Erratum: Magnetic and charge susceptibilities in the half-filled triangular lattice Hubbard model [Phys. Rev. Research 2, 013295 (2020)]
}

\author{
Shaozhi Liø and Emanuel Gull
}

(Received 13 July 2021; published 21 July 2021)

DOI: 10.1103/PhysRevResearch.3.039001

There is an error in the static magnetic susceptibility from dynamical mean-field theory (DMFT) shown in Fig. 1 of Phys. Rev. Research 2, 013295 (2020), where DMFT results are compared to the ladder-dual fermion approach (LDFA) results.

Figure 1 shows the static magnetic susceptibility $\chi_{m}\left(\mathbf{q}, i v_{0}\right)$ of DMFT (left panel) and LDFA (right panel) for $U=6 t$ and $T=t / 6$. The DMFT results previously shown indicated that $\chi_{m}\left(\mathbf{q}, i v_{0}\right)$ is negative near the $K$ point, implying the formation of a magnetically ordered state. This result is not correct. Figure E1 presents corrected DMFT results. At $U=6 t$, the corrected results have larger intensities near the $K$ point compared to the LDFA results. Our results show that the nonlocal correlation included in the LDFA weakens spin fluctuations and suppresses the magnetic phase transition.

We presented DMFT results in Fig. 1 in Sec. II B of the paper. The description of Fig. 1 in the paper should be changed to "Figure 1 shows $\chi_{m}\left(\mathbf{q}, i \nu_{0}\right)$ of DMFT (left panel) and LDFA (right panel) at $U=6 t$ and $\beta=6 / t$. DMFT results have larger intensities near the $K$ point compared to the LDFA results. Our results show that the nonlocal correlation included in the LDFA weakens spin fluctuations and suppresses the magnetic phase transition."

All other results are independent of the DMFT results presented in Fig. 1 and remain valid. None of our conclusions change.

We thank Thomas Schäfer for bringing this mistake to our attention.
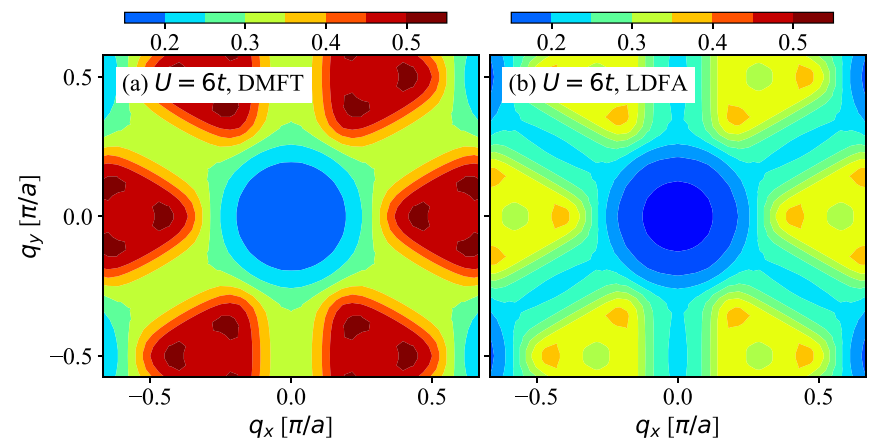

FIG. E1. Static magnetic susceptibility in momentum space $\chi_{m}\left(\mathbf{q}, i v_{0}\right)$ for $U=6 t$ at $T=t / 6$. The left (right) panel shows results of DMFT (LDFA).

Published by the American Physical Society under the terms of the Creative Commons Attribution 4.0 International license. Further distribution of this work must maintain attribution to the author(s) and the published article's title, journal citation, and DOI. 